\title{
Core components of bacterial protein secretion systems revealed at high resolution by cryo-electron microscopy
}

Justin C. Deme ${ }^{1,3}$, Lucas Kuhlen ${ }^{1}$, Patrizia Abrusci ${ }^{1}$, Steven Johnson ${ }^{1}$, Frederic Lauber ${ }^{2}$, Ben C. Berks ${ }^{2}$, Susan M. Lea ${ }^{1,3}$

${ }^{1}$ Sir William Dunn School of Pathology, University of Oxford, South Parks Road, Oxford OX1 3RE, United Kingdom

${ }^{2}$ Department of Biochemistry, University of Oxford, South Parks Road, Oxford OX1 3QU, United Kingdom

${ }^{3}$ Central Oxford Structural Microscopy and Imaging Centre, University of Oxford, South Parks Road, Oxford OX1 3RE, United Kingdom

Pathogenic bacteria use protein secretion systems for nutrient acquisition, motility, cell-to-cell communication, host defense, and delivery of virulence factors. Here novel, high resolution structures of core components of secretion systems are revealed by single particle reconstruction cryo-electron microscopy (cryo-EM), with emphasis on the Type III secretion system (T3SS) flagellar assembly apparatus. In T3SS, three putative integral membrane proteins (FliP/FliQ/FliR) are thought to form the core of an export gate formed in the cytoplasmic membrane. To date, their structure, assembly and location within the final nanomachine has remained elusive. Structures of several homologs of this complex resolved to $4.2 \AA$ by cryo-EM will be presented. These subunits complex to form a supra-membrane helical assembly with 5:4:1 stoichiometry. Unexpectedly, fitting these structures into reconstructions of intact secretion systems localize the export gate as a central component of the periplasmic portion of the machinery. This work further identifies the export gate as a core element of the secretion channel, making it an attractive target for antibiotic development. 